\title{
Multiple coexisting states of liquid rope coiling
}

\author{
By N. M. RIBE ${ }^{1}$, H. E. HUPPER T ${ }^{2}$, M. A. HALLWORTH ${ }^{2}$, \\ M. HAB IB I I ${ }^{3,4}$ AND D A NIEL B O N N $\mathbf{N}^{4,5}$
}

\footnotetext{
${ }^{1}$ Institut de Physique du Globe, UMR 7154 CNRS, 4 place Jussieu, 75252 Paris cédex 05, France

${ }^{2}$ Institute of Theoretical Geophysics, Department of Applied Mathematics and Theoretical Physics, University of Cambridge, Wilberforce Road, Cambridge CB3 0WA, UK

${ }^{3}$ Institute for Advanced Studies in Basic Sciences, Zanjan 45195-1159, Iran

${ }^{4}$ Laboratoire de Physique Statistique, UMR 8550 CNRS, Ecole Normale Supérieure, 24, rue Lhomond, 75231 Paris cédex 05, France

${ }^{5}$ Van der Waals-Zeeman Institute, University of Amsterdam, Valckenierstraat 65, 1018 XE Amsterdam, The Netherlands
}

(Received 25 February 2005 and in revised form 21 October 2005)

A thin 'rope' of viscous fluid falling from a sufficient height onto a surface forms a series of regular coils. Here we investigate theoretically and experimentally a curious feature of this instability: the existence of multiple states with different frequencies at a fixed value of the fall height. Using a numerical model based on asymptotic 'thin rope' theory, we determine curves of coiling frequency $\Omega$ vs. fall height $H$ as functions of the fluid viscosity $v$, the diameter $d$ of the injection hole, the volumetric injection rate $Q$, and the acceleration due to gravity $g$. In addition to the three coiling modes previously identified (viscous, gravitational and inertial), we find a new multivalued 'inertio-gravitational' mode that occurs at heights intermediate between gravitational and inertial coiling. In the limit when the rope is strongly stretched by gravity and $\Pi_{1} \equiv\left(v^{5} / g Q^{3}\right)^{1 / 5} \gg 1$, inertio-gravititational coiling occurs in the height range $O\left(\Pi_{1}^{-1 / 6}\right) \leqslant H\left(g / v^{2}\right)^{1 / 3} \leqslant O\left(\Pi_{1}^{-5 / 48}\right)$. The frequencies of the individual branches are proportional to $(g / H)^{1 / 2}$, and agree closely with the eigenfrequencies of a whirling liquid string with negligible resistance to bending and twisting. The number of coexisting branches scales as $\Pi_{1}^{5 / 32}$. The predictions of the numerical model are in excellent agreement with laboratory experiments performed by two independent groups using different apparatus and working fluids. The experiments further show that interbranch transitions in the inertio-gravitational regime occur via an intermediate state with a 'figure of eight' geometry that changes the sense of rotation of the coiling.

\section{Introduction}

A thin stream of honey poured from a sufficient height forms a regular helical coil as it approaches a piece of toast. This instability was called 'liquid rope coiling' by Barnes \& Woodcock (1958), whose pioneering work was the first in a series of experimental studies spanning nearly 50 years (Barnes \& Woodcock 1958; Barnes \& MacKenzie 1959; Cruickshank 1980; Cruickshank \& Munson 1981; Huppert 1986; Griffiths \& Turner 1988; Mahadevan, Ryu \& Samuel 1998; Maleki et al. 2004). Figure 1 shows the set-up used in most of these experiments, in which fluid with density $\rho$, viscosity $v$ and surface tension coefficient $\gamma$ is injected at a volumetric rate $Q$ from a hole of diameter $d \equiv 2 a_{0}$ and then falls a distance $H$ onto a solid surface. In general, the rope comprises a long nearly vertical 'tail' and a helical 'coil' of radius $R$ near the plate. 


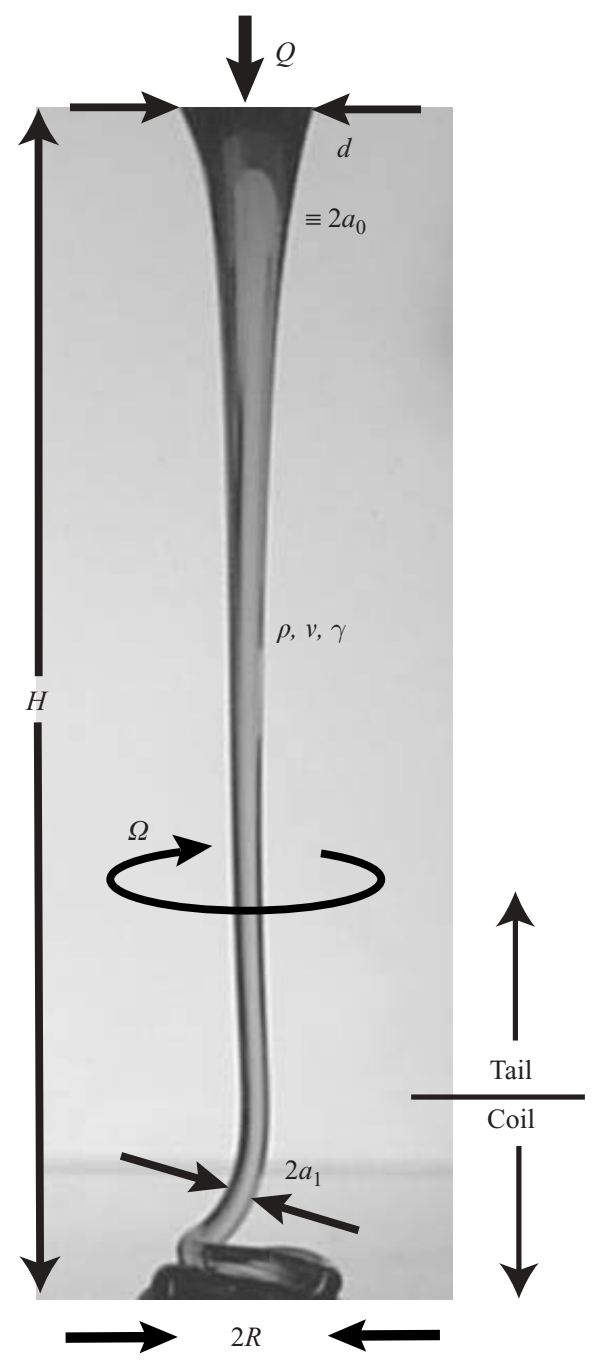

FIGURE 1. Steady coiling of a 'rope' of viscous corn syrup (photograph by N. Ribe.) Fluid with density $\rho$, viscosity $v$ and surface tension coefficient $\gamma$ is injected at volumetric rate $Q$ through a hole of diameter $d \equiv 2 a_{0}$ and falls a distance $H$ onto a solid surface. The radius of the rope at its point of contact with the plate is $a_{1}$, the angular coiling frequency is $\Omega$, and the radius of the circle along which the rope is laid down is $R$.

The first important advance towards a theoretical understanding of liquid rope coiling was Taylor's (1968) recognition that the phenomenon is fundamentally a buckling instability that requires a longitudinal compressive stress, like the buckling of an elastic column under a load. Subsequently, the critical fall height and frequency at the onset of coiling were determined using linear stability analysis (Cruickshank 1988; Tchavdarov, Yarin \& Radev 1993). Finite-amplitude coiling was first successfully explained by Mahadevan, Ryu \& Samuel (2000), who showed that rapid coiling is governed by a balance between rotational inertia and viscous forces. More recent studies, however, have shown that this scaling is only one among several possibilities. Using a numerical model for a thin liquid rope, Ribe (2004) showed that coiling can occur in three distinct dynamical regimes - viscous, gravitational, and inertial - depending on 
how the viscous forces in the helical coil are balanced. The existence of these three regimes was demonstrated experimentally by Maleki et al. (2004).

Here we study what is surely the most curious and complex feature of liquid rope coiling: the existence of multiple states with different frequencies at a fixed value of the input parameters. Ribe (2004) predicted that multivalued curves of frequency vs. height should be observed when coiling occurs in a gravitational-to-inertial transitional regime corresponding to intermediate fall heights. Subsequently, Maleki et al. (2004) observed an oscillation between two frequencies at a fixed fall height that corresponded closely to the numerical predictions. This phenomenon is reminiscent of one observed in (hitherto unpublished) experiments performed at the University of Cambridge in 1986, which showed that steady coiling becomes time-dependent at a critical value of the fall height. We demonstrate below that the time-dependence observed in the Cambridge experiments and the multivaluedness documented by Ribe (2004) and Maleki et al. (2004) are the same phenomenon. First, however, we review briefly the broader context in which it occurs.

The dynamical regime in which coiling takes place is determined by the magnitudes of the viscous $\left(F_{V}\right)$, gravitational $\left(F_{G}\right)$ and inertial $\left(F_{I}\right)$ forces per unit rope length within the coil. These are (Mahadevan et al. 2000; Ribe 2004)

$$
F_{V} \sim \rho v a_{1}^{4} U_{1} R^{-4}, \quad F_{G} \sim \rho g a_{1}^{2}, \quad F_{I} \sim \rho a_{1}^{2} U_{1}^{2} R^{-1},
$$

where $a_{1}$ is the radius of the rope within the coil and $U_{1} \equiv Q / \pi a_{1}^{2}$ is the corresponding axial velocity of the fluid. Because the rope radius is nearly constant in the coil, we define $a_{1}$ to be the radius at the point of contact with the plate. Each of the forces (1.1) depends strongly on $a_{1}$, which in turn is determined by the amount of gravity-induced stretching that occurs in the tail. Because this stretching increases strongly with the height $H$, the relative magnitudes of the forces $F_{V}, F_{G}$ and $F_{I}$ are themselves functions of $H$. As $H$ increases, the coiling traverses a series of distinct dynamical regimes characterized by different force balances in the coil. Figure 2 shows how these regimes are reflected in curves of $\Omega(H)$ and $a_{1}(H)$ for the parameters of one of the laboratory experiments reported by Maleki et al. (2004). These curves were determined by solving numerically the thin-rope equations of Ribe (2004) using the code AUTO 97 (Doedel et al. 2002). For simplicity, we neglected surface tension, which typically modifies the coiling frequency by no more than a few per cent for a surface tension coefficient $\gamma \approx 22 \mathrm{dyn} \mathrm{cm}^{-1}$ typical of silicone oil. Surface tension is, however, important in related phenomena such as the thermal bending of liquid jets by Marangoni stresses (Brenner \& Parachuri 2003).

For small heights $H\left(g / v^{2}\right)^{1 / 3}<0.08$, coiling occurs in the viscous (V) regime, in which both gravity and inertia are negligible and the net viscous force on each fluid element is zero. The coiling frequency decreases strongly with height, and the rope radius is nearly constant because no gravity-induced stretching occurs - indeed, the rope is slightly compressed against the plate $\left(a_{1}>a_{0}\right)$ for $H\left(g / v^{2}\right)^{1 / 3} \leqslant 0.06$. Because the fluid velocity is determined kinematically by the injection speed, the coiling frequency is independent of viscosity and is proportional to (Ribe 2004)

$$
\Omega_{V}=\frac{Q}{H a_{1}^{2}} .
$$

For $0.08 \leqslant H\left(g / v^{2}\right)^{1 / 3} \leqslant 0.4$, viscous forces in the coil are balanced by gravity $\left(F_{G} \approx F_{V} \gg F_{I}\right)$, giving rise to gravitational $(\mathrm{G})$ coiling with a frequency proportional to (Ribe 2004)

$$
\Omega_{G}=\left(\frac{g Q^{3}}{v a_{1}^{8}}\right)^{1 / 4},
$$




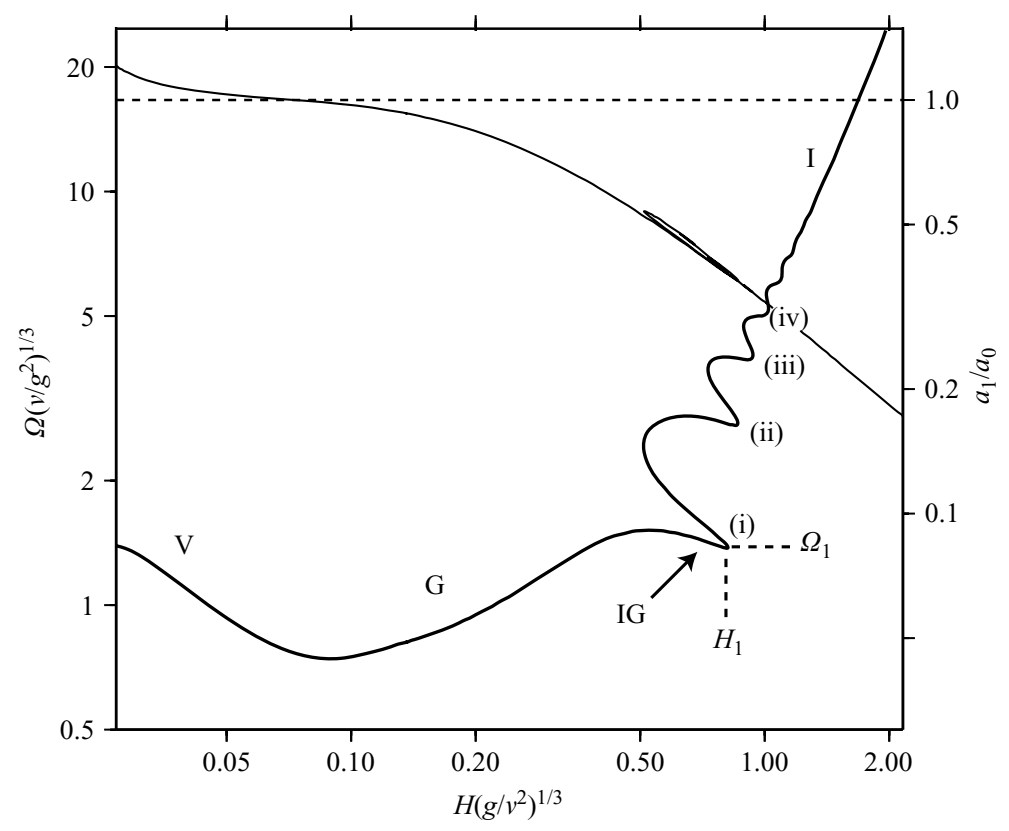

FIGURE 2. Dimensionless coiling frequency $\Omega\left(v / g^{2}\right)^{1 / 3}$ (heavy solid line, left-hand scale) and rope radius $a_{1} / a_{0}$ (light solid line, right-hand scale) as a function of dimensionless fall height $H\left(g / v^{2}\right)^{1 / 3}$, predicted numerically for $\Pi_{1}=7142$ and $\Pi_{2}=3.67$. Dimensionless groups $\Pi_{1}$ and $\Pi_{2}$ are defined by (2.1). Dashed line at $a_{1} / a_{0}=1$ is for reference. Portions of the heavy solid line representing the different coiling regimes are labelled: viscous (V), gravitational $(\mathrm{G})$, inertio-gravitational (IG), and inertial (I). Four turning points in the curve of frequency $v s$. height are indicated, and the height and frequency of the first are denoted $H_{1}$ and $\Omega_{1}$, respectively.

which is identical to the frequency scale for folding of a rope confined to a plane (Skorobogatiy \& Mahadevan 2000). The rope's radius is nearly constant $\left(a_{1} \approx a_{0}\right)$ at the lower end $\left(0.08 \leqslant H\left(g / \nu^{2}\right)^{1 / 3} \leqslant 0.15\right)$ of the gravitational regime, implying the seemingly paradoxical conclusion that gravitational stretching in the tail can be negligible in 'gravitational' coiling. The paradox is resolved by noting that for a given strain rate, the viscous forces associated with bending and twisting of a slender rope are much smaller than those that accompany stretching. The influence of gravity is therefore felt first in the (bending/twisting) coil and only later in the (stretching) tail, and thus can be simultaneously dominant in the former and negligible in the latter.

For $0.4 \leqslant H\left(g / v^{2}\right)^{1 / 3} \leqslant 1.2$, viscous forces in the coil are balanced by both gravity and inertia, giving rise to a complex transitional regime. The curve of frequency $v$ s. height is now multivalued, comprising a series of roughly horizontal 'steps' connected by 'switchbacks' with strong negative slopes. The curve exhibits four turning points (labelled (i)-(iv) in figure 2) where it folds back on itself. The additional 'wiggles' at larger values of $H$ are not turning points because the slope of the curve is always positive. For the example of figure 2, up to five frequencies are possible at a given height. Near the turning points, the frequency obeys a new 'inertio-gravitational' (IG) scaling, the expression for which we determine below. The amplitude of the folds in the curve of frequency $v$ s. height gradually decreases until the curve becomes smooth again at $H\left(g / v^{2}\right)^{1 / 3} \approx 1.2$. Viscous forces in the coil are now balanced almost entirely by inertia $\left(F_{I} \approx F_{V} \gg F_{G}\right)$, giving rise to inertial (I) coiling with a frequency 
proportional to (Mahadevan et al. 2000)

$$
\Omega_{I}=\left(\frac{Q^{4}}{v a_{1}^{10}}\right)^{1 / 3}
$$

The rope radius $a_{1}$, unlike the frequency, decreases monotonically with height over the entire height range, and is single-valued apart from a slight multiplicity in the range $0.5 \leqslant H\left(g / v^{2}\right)^{1 / 3} \leqslant 1.2$ where $\Omega(H)$ itself is multivalued. The decrease of $a_{1}$ is due to gravitational stretching of the tail with negligible vertical inertia, even in the 'inertial' regime $1.2 \leqslant H\left(g / v^{2}\right)^{1 / 3} \leqslant 2$. Again, the apparent paradox is resolved by noting that inertia, like gravity, can be simultaneously dominant in the coil and negligible in the tail. Vertical inertia eventually becomes important in the tail as well when $H\left(g / v^{2}\right)^{1 / 3}$ exceeds a value $\approx 3$.

Our aim in the present study is to characterize systematically the multivalued regime of liquid rope coiling using a combination of numerical modelling and laboratory experiments. We first survey this regime numerically to generate maps of the height and frequency of the first turning point as functions of the flow rate, the rope's initial radius, and the fluid viscosity. We then identify a new 'inertio-gravitational' mode within the multivalued regime and determine the scaling laws it obeys. Finally, we compare the predictions of the numerical model with laboratory experiments performed by two different groups using different apparatus and working fluids.

\section{Cartography of multivalued coiling}

We begin by determining the conditions under which the curve $\Omega(H)$ is multivalued. In a typical laboratory experiment, the fall height $H$ is varied while the hole diameter $d$, the flow rate $Q$, the viscosity $v$, and the acceleration due to gravity $g$ are held fixed. In the absence of surface tension, therefore, a given experiment can be characterized by the two dimensionless groups

$$
\Pi_{1}=\left(\frac{v^{5}}{g Q^{3}}\right)^{1 / 5}, \quad \Pi_{2}=\left(\frac{v Q}{g d^{4}}\right)^{1 / 4} .
$$

Each pair of values $\left(\Pi_{1}, \Pi_{2}\right)$ is associated with a different curve of frequency $v$ s. height. A numerical survey of the $\left(\Pi_{1}, \Pi_{2}\right)$-plane shows that $\Omega(H)$ is multivalued only if $\Pi_{1}$ exceeds a critical value $\Pi_{1}^{\text {crit }}$. Figure $3(a)$ shows the critical curve $\Pi_{1}^{\text {crit }}\left(\Pi_{2}\right)$ and figures $3(b)$ and $3(c)$ show curves of frequency vs. height for selected reference values (denoted A-D) of $\Pi_{1}$ and $\Pi_{2}$. The character of the transition from single-valued to multi-valued coiling is evident from the curves for points $\mathrm{A}, \mathrm{B}$ and $\mathrm{C}$, which all have $\Pi_{1}=10^{4}$. Just above the critical curve (point $\mathrm{A}$ ), $\Omega(H)$ is single-valued, but exhibits incipient 'wiggles'. As $\Pi_{2}$ decreases (points B and C), the curve is progressively compressed in the horizontal direction, leading to the appearance of folds and multivaluedness. For the range of $\Pi_{1}$ shown in figure 3 , the maximum number of turning points is four, and of coexisting states, five (e.g. at point C). However, these numbers increase without limit as $\Pi_{1} \rightarrow \infty$, as we shall demonstrate below.

Next, we map the height $H_{1}$ and the frequency $\Omega_{1}$ of the first turning point as functions of $\Pi_{1}$ and $\Pi_{2}$. The dynamical importance of the first turning point is revealed by laboratory experiments which show that when $H \approx H_{1}$, coiling oscillates between a state with frequency $\Omega_{1}$ and a state with a higher frequency (Maleki et al. 2004). 

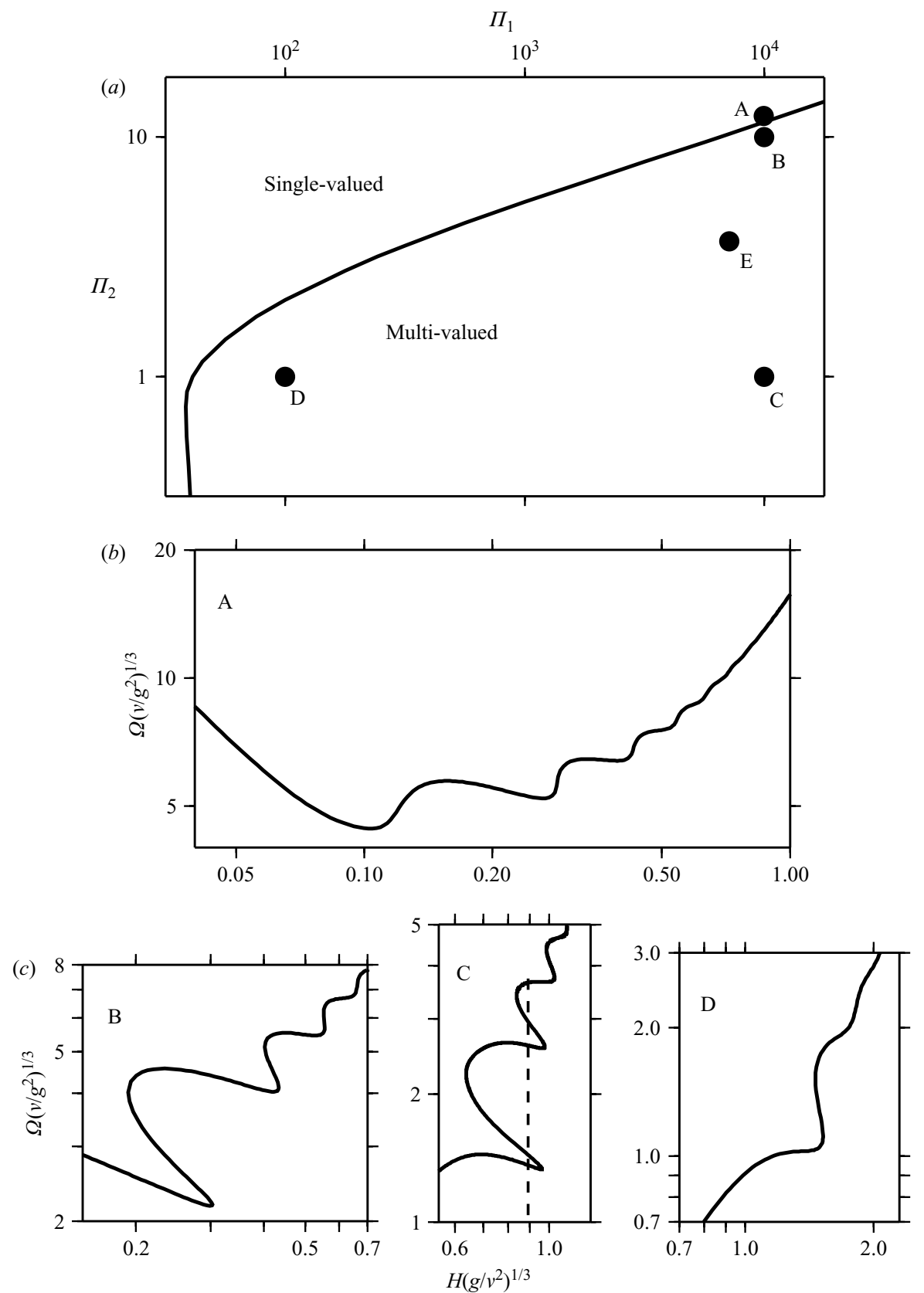

FIgURE 3. (a) Critical curve for the onset of multivalued coiling in the $\left(\Pi_{1}, \Pi_{2}\right)$-plane. The function $\Omega(H)$ is single-valued above/to the left of the curve. Points $\mathrm{A}-\mathrm{D}$ are reference points, and point $\mathrm{E}$ shows the values of $\Pi_{1}$ and $\Pi_{2}$ used in figure 2. $(b-c)$ Curves of frequency vs. height for the reference points $\mathrm{A}-\mathrm{D}$ in $(a)$. The dashed line in panel $\mathrm{C}$ is at a value of $H\left(g / v^{2}\right)^{1 / 3}$ for which 5 distinct coiling states exist.

Standard dimensional analysis requires

$$
H_{1}=\left(\frac{v^{2}}{g}\right)^{1 / 3} G_{1}\left(\Pi_{1}, \Pi_{2}\right), \quad \Omega_{1}=\left(\frac{g^{2}}{v}\right)^{1 / 3} G_{2}\left(\Pi_{1}, \Pi_{2}\right),
$$



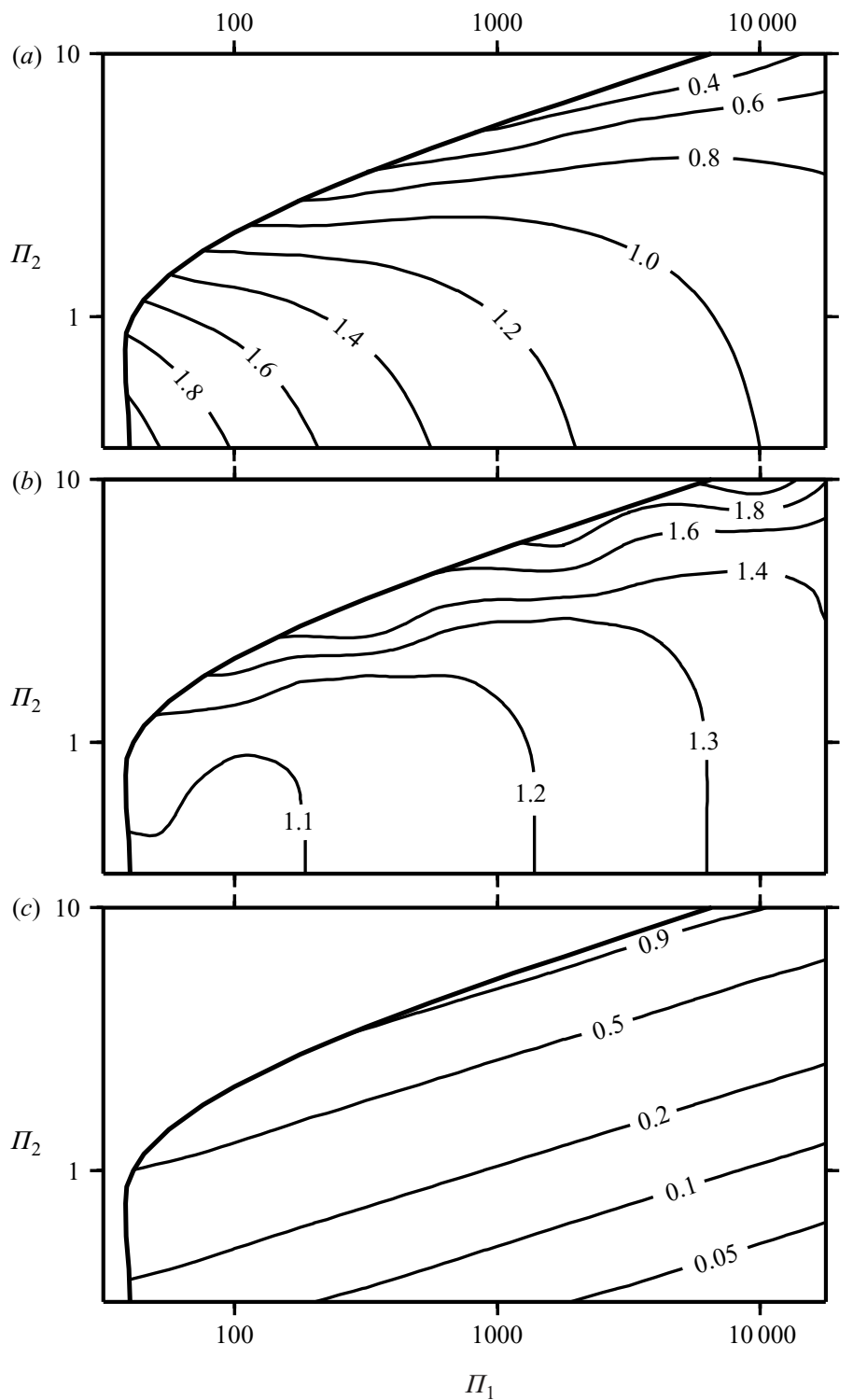

Figure 4. Contours of $(a)$ height $H_{1}\left(g / v^{2}\right)^{1 / 3},(b)$ frequency $\Omega_{1}\left(v / g^{2}\right)^{1 / 3}$, and $(c)$ thinning factor $a_{1} / a_{0}$ at the first turning point in the curve of frequency vs. height. Dimensionless groups $\Pi_{1}$ and $\Pi_{2}$ are defined by (2.1).

where $G_{1}$ and $G_{2}$ are unknown functions. These were calculated numerically, and are shown in figure 4. Also shown for reference (figure $4 c$ ) is the 'thinning factor' $a_{1} / a_{0}$ that measures the total amount of gravity-induced stretching in the tail.

The maps of figure 4 have simpler forms in the limit of strong gravitational stretching $\left(a_{1} / a_{0} \ll 1\right)$, which obtains when the 'buoyancy number' $B \equiv \pi a_{0}^{2} g H^{2} / \nu Q>$ 300 (Ribe 2004). This limit corresponds to the lower right-hand portion of the $\left(\Pi_{1}\right.$, $\Pi_{2}$ )-plane (figure $4 c$ ). Coiling in this limit is not influenced by the diameter $d$ of the injection hole. Accordingly, a (properly scaled) curve of coiling frequency vs. fall height will depend only on $\Pi_{1}$, and not on $\Pi_{2} \equiv(\nu Q / g)^{1 / 4} d^{-1}$. This is demonstrated 


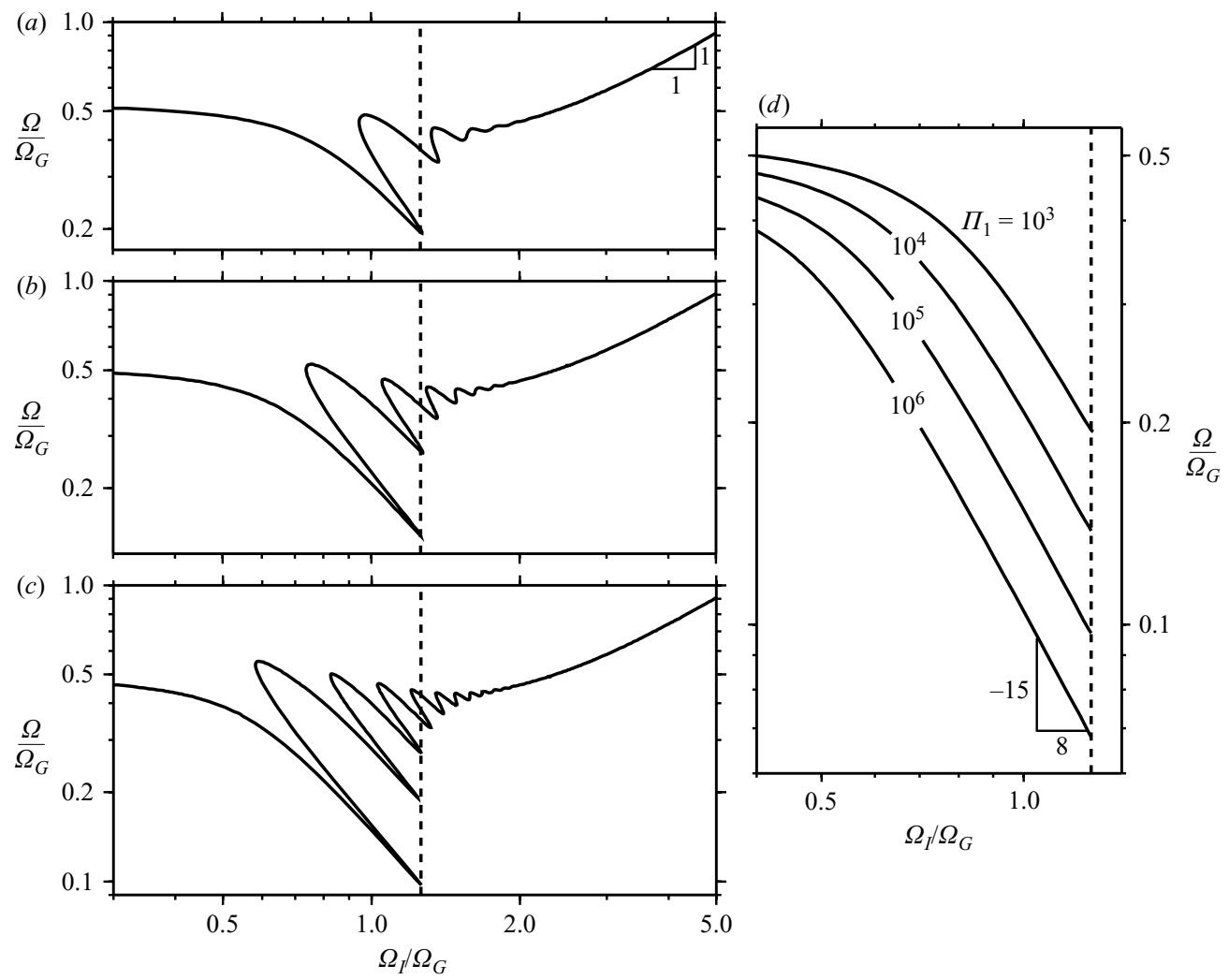

FIGURE 5. Scaled coiling frequency $\Omega / \Omega_{G}$ as a function of $\Omega_{I} / \Omega_{G}$, for $(a)\left(\Pi_{1}, \Pi_{2}\right)=$ $\left(10^{3}, 0.316\right),(b)\left(10^{4}, 0.316\right)$, and $(c)\left(10^{5}, 0.562\right)$. Leftmost (slope $\left.=0\right)$ and rightmost (slope $\left.=1\right)$ portions of the curves correspond to gravitational and inertial coiling, respectively. $(d)$ The lower branches of the portions of the curves that lie to the left of the first turning point shown in greater detail. The curve for $\Pi_{1}=10^{6}$ has $\Pi_{2}=0.316$. The vertical dashed lines are at $\Omega_{I} / \Omega_{G}=1.26$.

in figures 5(a)-5(c), which show $\log -\log$ plots of the scaled coiling frequency $\Omega / \Omega_{G}$ vs. $\Omega_{I} / \Omega_{G}$ for three values of $\Pi_{1}$. On each of these plots, purely gravitational coiling $\left(\Omega \propto \Omega_{G}\right)$ appears as a segment with zero slope at the left-hand side $\left(\Omega_{I} / \Omega_{G} \ll 1\right)$, and purely inertial coiling $\left(\Omega \propto \Omega_{I}\right)$ as a segment with unit slope at the right-hand side $\left(\Omega_{I} / \Omega_{G} \gg 1\right.$.) Because $\Omega_{G} / \Omega_{I} \propto a_{1}^{-4 / 3}$ and $a_{1}$ decreases with $H$ (figure 2 ), the ratio $\Omega_{I} / \Omega_{G}$ can be thought of as a scaled fall height. A direct numerical calculation shows that curves of $\Omega / \Omega_{G}$ vs. $\Omega_{I} / \Omega_{G}$ for different values of $\Pi_{2}$ are indistinguishable from those shown in figures $5(a)-(c)$, as long as $a_{1} / a_{0} \leqslant 0.1$.

\section{Inertio-gravitational regime}

The shapes of the curves shown in figure 5 differ significantly in the middle range $0.6 \leqslant \Omega_{I} / \Omega_{G} \leqslant 2$, where both the number and amplitude of the oscillations increase with increasing $\Pi_{1}$. However, a more detailed examination (figure $5 d$ ) reveals a universal structure: the first turning point occurs at a nearly constant value of $\Omega_{I} / \Omega_{G} \approx 1.26$, and the curve approaching it is a power law with slope $-1.87 \approx-15 / 8$. These features indicate the existence of a new 'inertio-gravitational' coiling regime, whose nature we now investigate. 

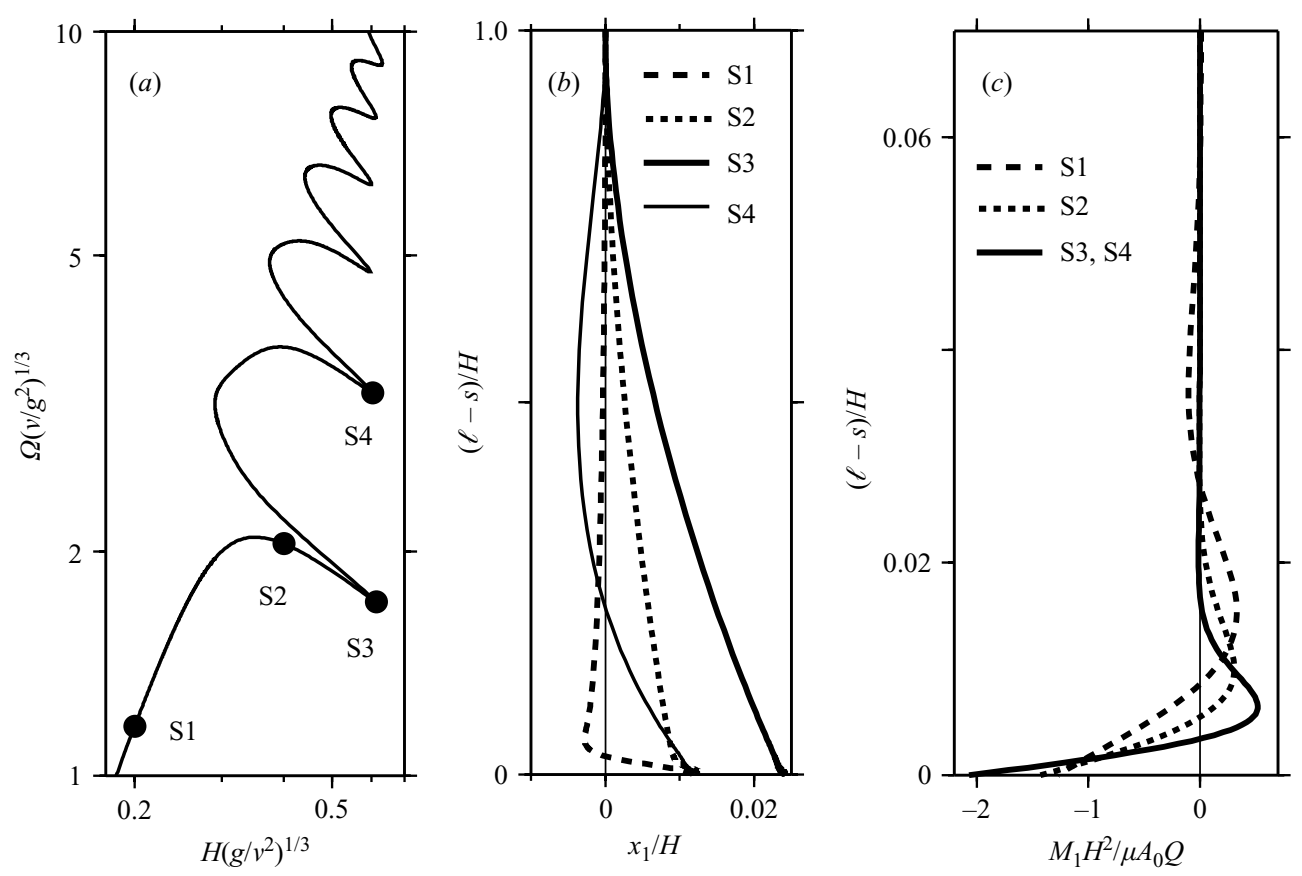

FIGURE 6. Structure of numerical solutions for steady coiling with $\Pi_{1}=10^{6}$ and $\Pi_{2}=0.316$. (a) Coiling frequency $\Omega$ vs. fall height $H$. Points S1-S4 denote selected numerical solutions. (b) Coordinate $x_{1}$ of the rope axis in the plane containing its two endpoints, for solutions S1-S4. The quantity $\ell-s$ is the arclength measured backwards from the contact point $s=\ell$. Because the rope is nearly vertical in all cases, $\ell \approx H$. (c) Same as $(b)$, but for the bending moment $M_{1}$, with $A_{0}=\pi a_{0}^{2}$. The curves for solutions S3 and S4 are identical to within the plotting line width.

The character of the new regime is revealed most clearly by the shape of the coiling rope. Figure $6(b)$ shows the lateral displacement $x_{1}(s)$ of the rope in the plane containing the injection point and the contact point, for four numerical solutions with $\Pi_{1}=10^{6}, \Pi_{2}=0.316$, and different values of $H\left(g / v^{2}\right)^{1 / 3}$. These four solutions are denoted by the points S1-S4 on the corresponding curve of frequency vs. height (figure $6 a$ ). Figure $6(c)$ shows, for the same solutions, the moment $M_{1}(s)$ associated with bending about a local basis vector $\boldsymbol{d}_{1}$ that is normal to the axis of the rope (figure $7 b$ ). Solution S1 corresponds to pure gravitational coiling with negligible inertia. Here the rope is nearly vertical except in a thin boundary layer near the contact point $s=\ell$ where viscous forces associated with bending are significant. As $H$ increases (solutions S2 and S3), however, the displacement of the rope becomes significant along its whole length, even though bending is still confined to a thin boundary layer near $s=\ell$ (figure $6 c$ ).

A further important indication is obtained by comparing the structures of the numerical solutions at the first two turning points S3 and S4 (figure $6 a$ ). The solutions for $M_{1}(s)$ at points $\mathrm{S} 3$ and $\mathrm{S} 4$ are identical to within the width of the solid line in figure 6(c). The corresponding displacements $x_{1}(s)$ and frequencies $\Omega$, however, are quite different (figure $6 b$ ). It thus appears that the dynamics of this regime is controlled by the tail of the rope, and that the bending boundary layer plays a merely passive role. 


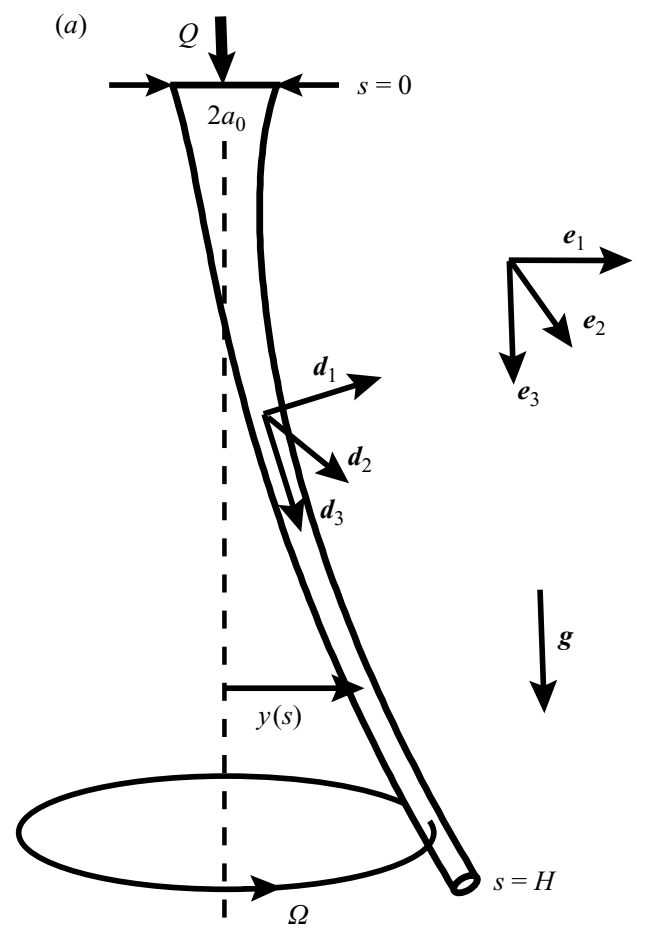

(b)

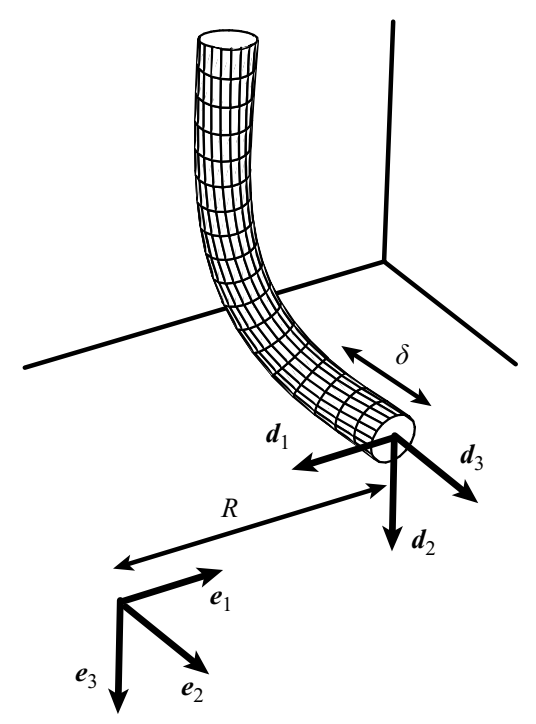

FIGURE 7. Geometry of liquid rope coiling in the inertio-gravitational regime. $\boldsymbol{e}_{i}(i=1,2,3)$ are Cartesian unit vectors fixed in a frame rotating with the rope, and $\boldsymbol{d}_{i}$ are orthogonal material unit vectors defined at each point on its axis, $\boldsymbol{d}_{3}$ being the tangent vector. The parameters $Q$, $a_{0}, H, R$ and $\Omega$ are defined as in figure 1. (a) Geometry of the tail, modelled as an extensible string with negligible resistance to bending and twisting. This string lies in the plane normal to $\boldsymbol{e}_{2}$, and $\boldsymbol{d}_{1} \cdot \boldsymbol{e}_{2}=0$. The lateral displacement of the axis from the vertical is $y(s)$, where $s$ is the arclength measured from the injection point. $(b)$ Geometry of the rope near the contact point. Bending and twisting are important in a boundary layer of arcwise extent $\delta$ near this point. Right at the contact, $\boldsymbol{d}_{1}$ is horizontal and points towards the center of the circle (of radius $R$ ) along which the rope is laid down.

We now demonstrate that the dynamics of the tail provide the key to explaining the multivaluedness of the frequency-height curve. Our numerical simulations show that the rates of viscous dissipation associated with bending and twisting in the tail are negligible compared to the dissipation rate associated with stretching. The tail can therefore be regarded as a 'liquid string' with negligible resistance to bending and twisting, whose motion is governed by a balance among gravity, the centrifugal force, and the axial tension associated with stretching. The balance of gravity and the centrifugal force normal to the tail requires

$$
\rho g A \sin \theta \sim \rho A \Omega^{2} y,
$$

where $A$ is the area of the cross-section of the tail, $\theta$ is its inclination from the vertical, and $y$ is the lateral displacement of its axis. Because $y \sim R$ and $\sin \theta \sim R / H,(3.1)$ implies that $\Omega$ is proportional to the scale

$$
\Omega_{I G}=\left(\frac{g}{H}\right)^{1 / 2},
$$

which is just the angular frequency of a simple pendulum. 


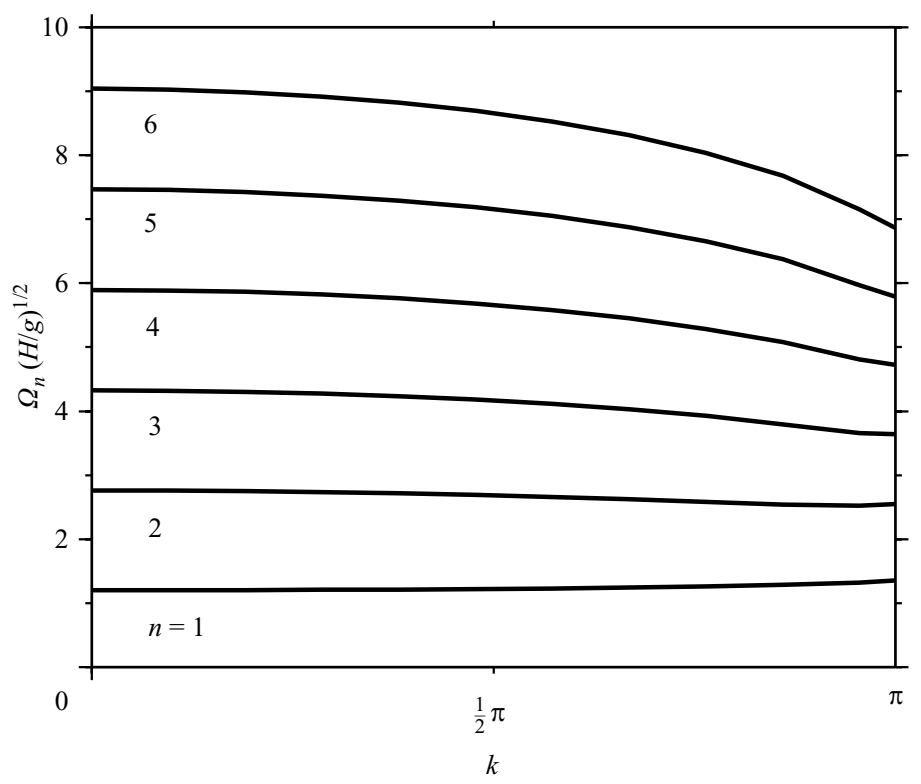

FigURE 8. First six eigenfrequencies $\Omega_{n}(k)$ of the boundary-value problem (3.3) for a whirling liquid string.

Further insight can be obtained by examining in more detail the dynamics of a whirling liquid string, a simple model of which is sketched in figure $7(a)$. In the Appendix, we show that the lateral displacement $y$ of the axis of the string satisfies the boundary-value problem

$$
k^{-1} \sin k(1-\tilde{s}) y^{\prime \prime}-y^{\prime}+\tilde{\Omega}^{2} y=0, \quad y(0)=0, \quad y(1) \text { finite, }
$$

where primes denote differentiation with respect to the dimensionless arclength $\tilde{s}=s / H$ and $\tilde{\Omega}=\Omega(H / g)^{1 / 2}$. The three terms in (3.3) represent the axis-normal components of the viscous, gravitational and centrifugal forces, respectively, per unit length of the string. The dimensionless parameter $k$ measures the degree of gravityinduced stretching of the string, and satisfies the transcendental equation

$$
0=2 B \cos ^{2} \frac{1}{2} k-3 k^{2}
$$

where $B$ is the buoyancy number defined earlier. The limit $k=0(B=0)$ corresponds to an unstretched string with constant radius, whereas a strongly stretched string has $k=\pi(B \rightarrow \infty)$.

Equations (3.3) define a boundary-eigenvalue problem which has non-trivial solutions only for particular values $\tilde{\Omega}_{n}(k)$ of the frequency $\tilde{\Omega}$. Figure 8 shows the first six of these eigenfrequencies as functions of $k$, determined using AUTO 97. In the limit $k=0$, we recover the classical solution for the eigenfrequencies of an inextensible chain, which satisfy $J_{0}\left(2 \tilde{\Omega}_{n}(0)\right)=0$, where $J_{0}$ is the Bessel function of the first kind of order 0 .

To test whether these eigenfrequencies correspond to the multiple frequencies seen in the full numerical solutions, we rescale the numerically predicted curves of frequency vs. height to curves of $\Omega / \Omega_{I G}$ vs. $\Omega_{G} / \Omega_{I G}$. On a $\log -\log$ plot, these rescaled curves should exhibit distinct segments with slopes of unity and zero, corresponding to gravitational $\left(\Omega \propto \Omega_{G}\right)$ and inertio-gravitational $\left(\Omega \propto \Omega_{I G}\right)$ coiling, respectively. Figure 9 shows $\Omega / \Omega_{I G}$ vs. $\Omega_{G} / \Omega_{I G}$ for $\Pi_{1}=10^{3}, 10^{5}$ and $10^{6}$. As expected, the 


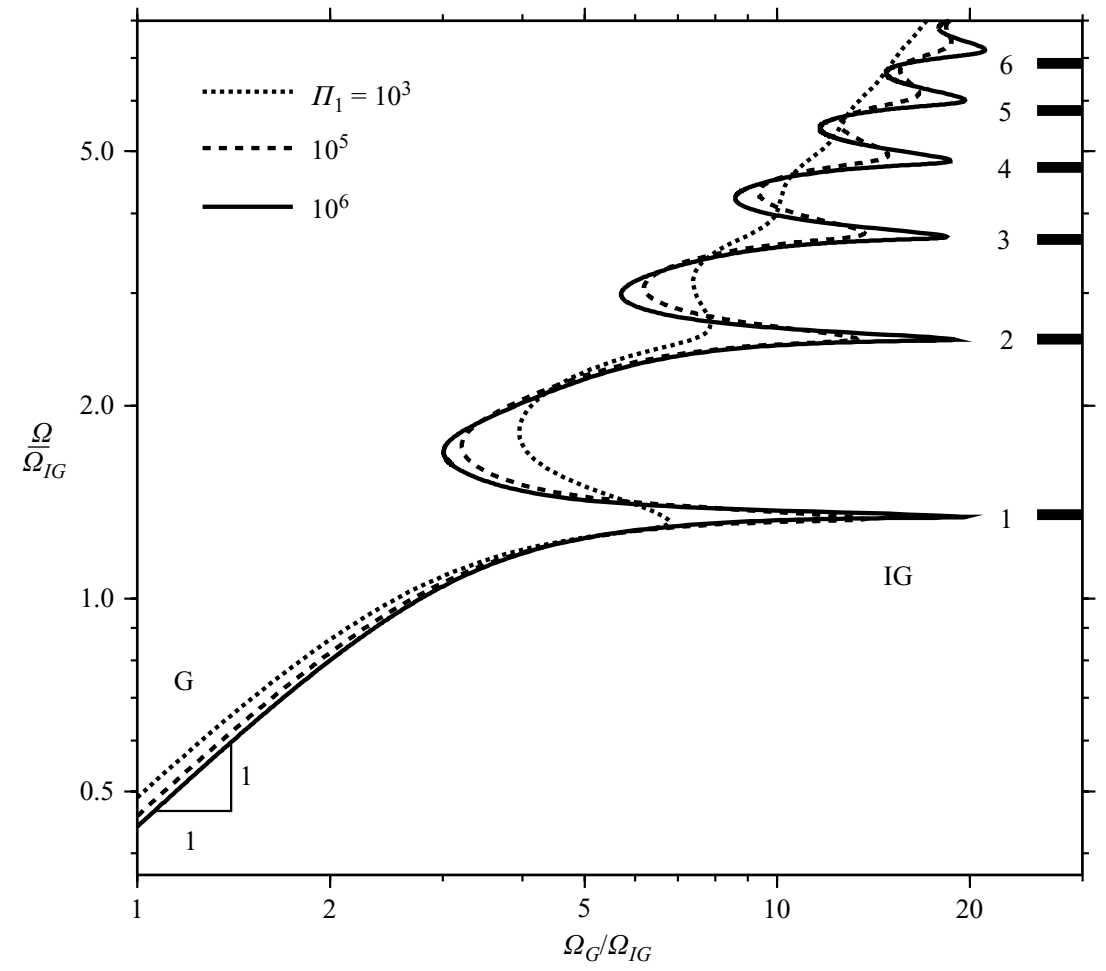

FIGURE 9. $\Omega / \Omega_{I G}$ vs. $\Omega_{G} / \Omega_{I G}$ in the limit of strong stretching $\left(a_{1} / a_{0} \ll 1\right)$ for $\Pi_{1}=10^{3}$ (dotted line), $10^{5}$ (dashed line), and $10^{6}$ (solid line.) The corresponding values of $\Pi_{2}$ are as in figure 5. Segments of the curves representing gravitational and inertio-gravitational coiling are denoted by $\mathrm{G}$ and IG, respectively. The horizontal black bars (right) indicate the first six eigenfrequencies of a strongly stretched $(k=\pi)$ whirling liquid string (figure 8).

rescaled curves clearly display a transition from gravitational coiling on the left-hand side to inertio-gravitational coiling on the right-hand side. Moreover, the multiple frequencies in the rescaled curves correspond very closely to the whirling string eigenfrequencies $\Omega_{n}(\pi)$ in the 'strong stretching' limit $k=\pi$, the first six of which are shown by the black bars at the right-hand side of figure 9 . We conclude that a rope coiling in the inertio-gravitational mode does indeed behave as a whirling liquid string with negligible resistance to bending and twisting.

The pendulum frequency scale (3.2) also explains why the slope of the curves of $\Omega / \Omega_{G}$ vs. $\Omega_{I} / \Omega_{G}$ is $-15 / 8$ in the inertio-gravitational regime (figure $5 d$ ). In this regime, the tail of the rope is stretched strongly $\left(a_{1} \ll a_{0}\right)$ by gravity, but the axial inertia $\rho A U U^{\prime}$ (unlike the centrifugal inertia $\rho A \Omega^{2} y$ ) is negligible there. Under these conditions, the radius $a_{1}$ of the stretched tail can be determined analytically, and is (Ribe 2004)

$$
a_{1}=\left(\frac{3 \pi \nu Q}{2 g}\right)^{1 / 2} H^{-1} \text {. }
$$

Upon using (3.5) to eliminate $H$ from the inertio-gravitational scaling law $\Omega \sim(g / H)^{1 / 2}$ and using the definitions of $\Omega_{G}, \Omega_{I}$ and $\Pi_{1}$, we obtain

$$
\frac{\Omega}{\Omega_{G}} \sim \Pi_{1}^{-5 / 32}\left(\frac{\Omega_{I}}{\Omega_{G}}\right)^{-15 / 8},
$$

in agreement with figure $5(d)$. 
While the whirling string model explains well the frequencies of the multiple solution branches, it cannot explain why these branches fold back on themselves at turning points. We now demonstrate that this behaviour is due to the influence of inertia in the bending boundary layer near the contact point. The geometry of this region is sketched in figure $7(b)$. Despite our previous usage, it is not quite accurate in this case to call the bending boundary layer a 'coil', because its arcwise extent $\delta$ is independent of the radius $R$ of the contact point. This is clear from figure 6, which shows that the numerical solutions at the first two turning points $\mathrm{S} 3$ and $\mathrm{S} 4$ have different values of $R \equiv x_{1}(H)$, but identical distributions of the bending moment $M_{1}(s)$. To estimate $\delta$, consider the balance of forces acting within the boundary layer in the $\boldsymbol{d}_{1}$-direction. Let the characteristic magnitudes of the viscous, gravitational and inertial forces per unit rope length be $F_{V}, F_{G}$ and $F_{I}$, respectively. The dominant force balance in the boundary layer is $F_{V} \sim F_{G}$, or (Ribe 2004)

$$
\rho v A^{2}\left(\kappa_{2} U\right)^{\prime \prime \prime} \sim \rho g A d_{13},
$$

where $d_{13}=\boldsymbol{d}_{1} \cdot \boldsymbol{e}_{3}$ and primes denote differentiation with respect to $s$. Within the boundary layer, $\mathrm{d} / \mathrm{d} s \sim \delta^{-1}, \kappa_{2} \sim R^{-1}$, and $d_{13} \sim \delta / R$. Moreover, in the limit of strong stretching $U \equiv Q / A \sim g H^{2} / \nu$. Finally, conservation of volume flux at the contact point requires $R \sim U / \Omega \sim H^{5 / 2} g^{1 / 2} / \nu$. Substitution of these expressions into (3.7) yields

$$
\delta \sim\left(\frac{\nu Q}{g}\right)^{1 / 4},
$$

which is identical to the scale for the coil radius $R$ in gravitational coiling (Ribe 2004). In view of (3.8) and the other expressions given above, the force balance (3.7) takes the form

$$
F_{V} \sim F_{G} \sim \rho\left(\frac{Q^{5} v^{9}}{g^{3} H^{9}}\right)^{1 / 4} .
$$

Now all three contributions (axial, centrifugal and Coriolis) to the inertial force within the boundary layer have the same order of magnitude $F_{I} \sim \rho A \kappa_{2} U^{2}$, or

$$
F_{I} \sim \rho Q\left(\frac{g}{H}\right)^{1 / 2} .
$$

By comparing (3.10) with (3.9), we see that inertia becomes comparable to the gravitational and viscous forces in the boundary layer when

$$
H \sim\left(\frac{\nu^{9} Q}{g^{5}}\right)^{1 / 16} .
$$

How is the height scale (3.11) related to the occurrence of turning points? To determine this, recall that the numerical solutions show that the height $H_{1}$ at which the first turning point occurs corresponds to a critical value of $\Omega_{I} / \Omega_{G}=1.26$ (figure 5.) By substituting into this expression the definitions (1.4) and (1.3) of $\Omega_{I}$ and $\Omega_{G}$ and then eliminating $a_{1}$ using (3.5), we obtain

$$
H_{1}=2.58\left(\frac{\nu^{9} Q}{g^{5}}\right)^{1 / 16} .
$$

The corresponding frequency $\Omega_{1} \equiv 1.34\left(\mathrm{~g} / H_{1}\right)^{1 / 2}$ is

$$
\Omega_{1}=0.834\left(\frac{g^{21}}{v^{9} Q}\right)^{1 / 32} .
$$




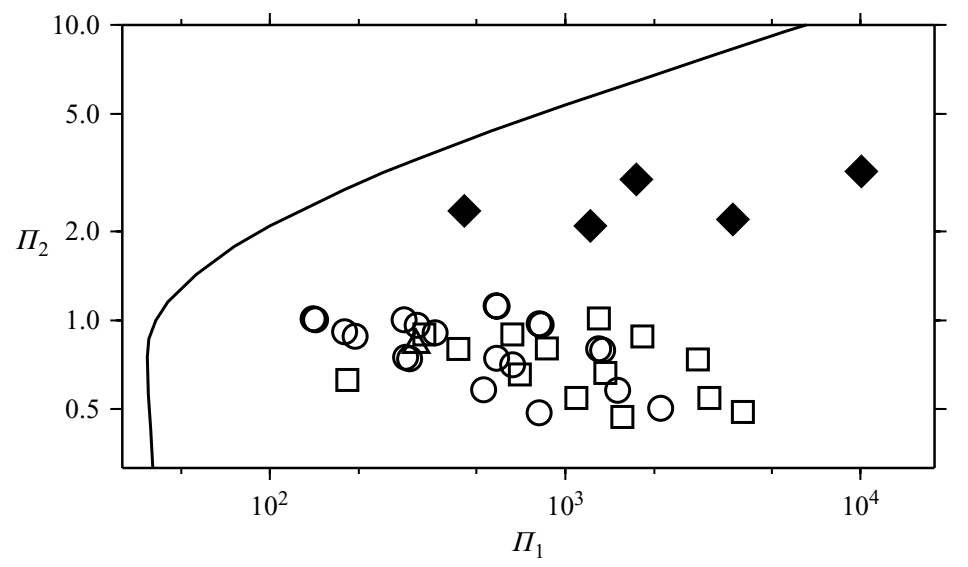

FIgURE 10. Distribution on the $\left(\Pi_{1}, \Pi_{2}\right)$-plane of the laboratory experiments discussed in this study. Symbols indicate experiments performed at Cambridge using Golden Syrup (open circles) and HYVIS-30 polybutene (open squares) and at Zanjan using silicone oil (solid diamonds.) The solid line is the critical curve for the onset of multivalued coiling from figure 3.

The equivalence of (3.12) and (3.11) suggests that turning points in the frequency $v s$. height curve occur when inertia becomes comparable to the viscous and gravitational forces in the bending boundary layer near the contact point.

The scaling laws (3.12) and (3.13) were derived under the assumption of strong stretching $\left(a_{1} / a_{0} \ll 1\right)$. Nevertheless, they give good approximations to the exact numerical results of figure 4 for values of $a_{1} / a_{0}$ that are not especially small. For $a_{1} / a_{0} \leqslant 0.25$, for example, the maximum errors of (3.12) and (3.13) are $15 \%$ and $5 \%$, respectively, when $\Pi_{1} \geqslant 100$. For $a_{1} / a_{0} \leqslant 0.10$, these errors decrease to $8 \%$ and $3 \%$, respectively.

\section{Comparison with experiment}

We now compare the predictions of the thin-rope numerical model with laboratory data obtained at the University of Cambridge (1986-1987) and at the Institute for Advanced Studies in Basic Sciences in Zanjan (2005.) Figure 10 shows the distribution of these experiments (40 in number) on the $\left(\Pi_{1}, \Pi_{2}\right)$-plane.

The Cambridge and Zanjan experiments differ in several important ways: the working fluids used, the injection method, and the data acquisition procedure. We present the two sets of experiments and their results in chronological order.

\subsection{The Cambridge experiments}

In the Cambridge experiments, Tate \& Lyle's Golden Syrup $\left(\rho=1.44 \mathrm{~g} \mathrm{~cm}^{-3}, v=\right.$ $\left.700 \mathrm{~cm}^{2} \mathrm{~s}^{-1}, \quad \gamma=78 \mathrm{dyn} \mathrm{cm}^{-1}\right)$ or HYVIS-30 polybutene $\left(\rho=0.90 \mathrm{~g} \mathrm{~cm}^{-3}, \quad v=\right.$ $1000 \mathrm{~cm}^{2} \mathrm{~s}^{-1}, \gamma=30 \mathrm{dyn}^{-1}$ ) was allowed to fall freely from a hole in the bottom of a reservoir onto a plate or (in some cases) a pool of the same fluid. The height of the plate was adjustable, and was read off to within $\pm 0.5 \mathrm{~mm}$ against a vertical scale. The flow rates for a given fluid and hole diameter were calibrated prior to each experiment by measuring the mass drained in a given time as a function of the depth of fluid in the reservoir. The coiling frequency was measured by using a stopwatch to record the time taken for a fixed number of revolutions (between 3 and 25.) As no high-speed imaging was available at the time, measurements were restricted to cases 

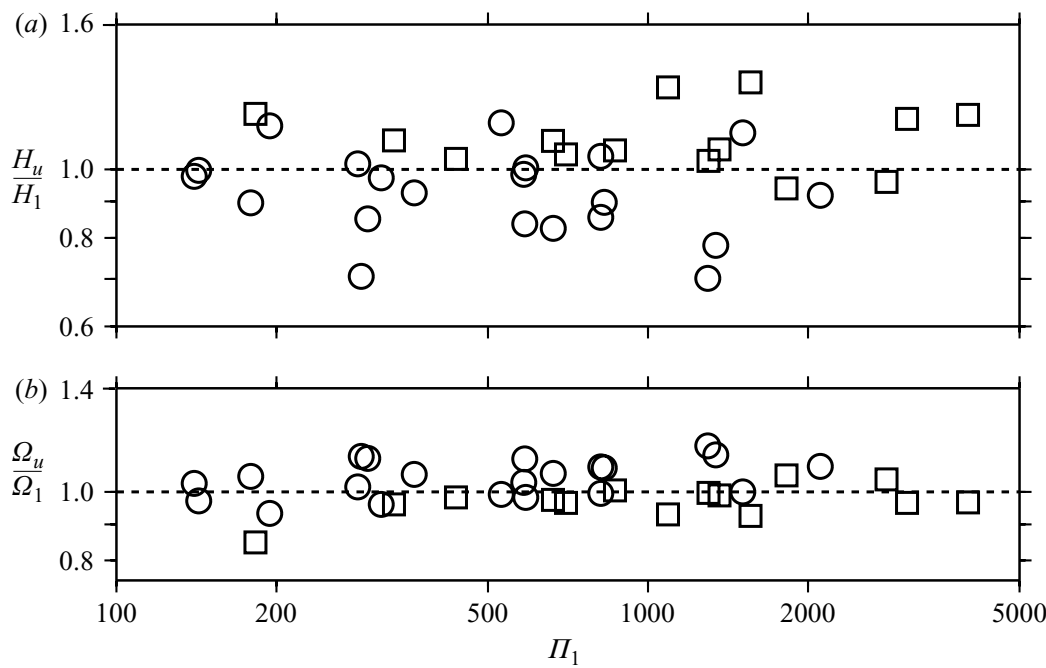

Figure 11. Comparison of the Cambridge experiments with numerical predictions of the thin-rope model. ( $a$ ) Ratio of the observed fall height $H_{u}$ at the onset of time dependence to the numerically predicted height $H_{1}$ of the first turning point. $(b)$ Same as $(a)$, but for the frequency $\Omega$. Symbols are the same as in figure 10 . The vertical scale is logarithmic. Root mean square errors for $H_{1}$ and $\Omega_{1}$ are $14 \%$ and $6 \%$, respectively. Error bars for $H_{u}$ are smaller than the plotting symbols, and those for $\Omega_{u}$ were not reported.

where individual revolutions could be tracked by eye. The fall height reported in each case was the distance from the hole in the reservoir to the plate, and was not corrected for the presence of piled-up fluid beneath the coiling rope. In each experiment, the fall height was gradually increased, and the critical height $H_{u}$ at which the coiling became unsteady was measured. The corresponding critical frequency $\Omega_{u}$, defined as the frequency of steady coiling at a height just below $H_{u}$, was taken as equal to the frequency measured at the last height before $H_{u}$.

Figure 11 compares these measured values to the numerically predicted height $H_{1}$ and frequency $\Omega_{1}$ of the first turning point in the curve of frequency vs. height for the same parameters. The effect of surface tension was included in these calculations. The predicted and observed heights differ by up to about $30 \%$, with an r.m.s. error of $14 \%$. The agreement of the frequencies is much better (r.m.s. error $\approx 7 \%$.) The poorer agreement in the former case may be due in part to the use of uncorrected values of the fall height. However, this would tend to make the ratio $H_{u} / H_{1}$ too large, and no such systematic bias is apparent in figure $11(a)$. We think that the more likely cause is the inherent structure of the multivalued function $\Omega(H)$, as will appear more clearly below. For now, we interpret the comparisons of figure 11 as strong evidence that the time-dependence observed in the Cambridge experiments reflects the multivaluedness of $\Omega(H)$ documented by Ribe (2004) and Maleki et al. (2004).

\subsection{The Zanjan experiments}

In the Zanjan experiments, silicone oil $\left(\rho=0.97 \mathrm{~g} \mathrm{~cm}^{-3}, v=125,300\right.$ or $1000 \mathrm{~cm}^{2} \mathrm{~s}^{-1}$, $\gamma=21.5 \mathrm{dyn}^{-1}$ ) was forcibly injected using a syringe pump controlled by a stepper motor. The flow rate $Q$ was determined to within $\pm 4.5 \%$ by recording the volume of fluid in the syringe as a function of time. This technique permitted access to portions of the $\left(\Pi_{1}, \Pi_{2}\right)$-plane that are hard to reach with free (gravity-driven) injection. The coiling frequency was determined by counting frames of movies taken with a 
videocamera ( 25 frames $\mathrm{s}^{-1}$.) For each point $\left(\Pi_{1}, \Pi_{2}\right)$ investigated, separate sets of measurements were obtained by increasing and decreasing the height over the range of interest, and in one case additional measurements were made at randomly chosen heights. The raw fall heights were corrected by subtracting the height of the pile of fluid on the plate beneath the coil. This ensures proper comparability with the numerical solutions, in which no pile forms because the fluid laid down on the plate is instantaneously removed. To avoid unintentional bias, the experiments were performed and the fall heights corrected before the corresponding curve of frequency $v$ s. height was calculated numerically. The effect of surface tension was included in all calculations.

A disadvantage of forced injection is that unwanted 'die-swell' occurs in some cases as the fluid exits the orifice. The radius of the tail then varies along the rope in a way significantly different from that predicted by our numerical model. Die-swell was negligible in all the experiments with $v=1000 \mathrm{~cm}^{2} \mathrm{~s}^{-1}$, but significant $(\approx 10-15 \%$ increase in radius) in some experiments performed with lower viscosities. Here we report only experiments for which die-swell did not exceed $10 \%$.

Figure 12 shows the coiling frequency $\Omega$ measured as a function of the corrected height $H$ for the five points $\left(\Pi_{1}, \Pi_{2}\right)$ shown as black diamonds in figure 10 , together with curves of $\Omega(H)$ predicted numerically for the same parameters. The observations and the numerical predictions agree extraordinarily well for experiments $(b)-(e)$, which were all performed with $v=1000 \mathrm{~cm}^{2} \mathrm{~s}^{-1}$. The somewhat poorer agreement for case (a) $\left(v=300 \mathrm{~cm}^{2} \mathrm{~s}^{-1}\right)$ is probably due to die-swell, which was about $10 \%$ in this experiment. The measurements are concentrated along the roughly horizontal steps of the numerically predicted curves, leaving the 'switchback' portions in between almost entirely empty. In all experiments, two coexisting coiling states with different frequencies exist over a small but finite range of fall heights; in experiment $(b)$, we observed three such states at $H \approx 10.8 \mathrm{~cm}$. In experiments $(a)-(d)$, the states observed along the first step in the curve extend right up to the first turning point. In experiment (e), by contrast, the coiling 'jumps' to the second step before the first turning point is reached.

Figure 12 suggests a likely reason why the critical heights observed in the Cambridge experiments (figure 11a) agree less well with the numerics than the critical frequencies do (figure 11b). First, the coexistence of two frequencies over a finite range of fall heights suggests that oscillation between the two may also occur over a range of heights. Yet because the steps in the curve $\Omega(H)$ are roughly horizontal, the frequency is almost independent of height in this range. The frequency of the onset of time-dependence should therefore exhibit much less variability than the height.

The Zanjan experiments also provide insight into the mechanism of the transition between coexisting coiling states. Figures $13(a)$ and 13(b) show the coil geometry for the states with lower and higher frequency that coexist at a total (uncorrected) fall height $H=7.1 \mathrm{~cm}$ with $v=1000 \mathrm{~cm}^{2} \mathrm{~s}^{-1}, d=0.068 \mathrm{~cm}$ and $Q=0.0042 \mathrm{~cm}^{3} \mathrm{~s}^{-1}$. Three significant differences between the two cases are apparent. The coil radius $R$ is smaller at the higher frequency, because $R=U_{1} / \Omega$ by definition and $U_{1}$ (which depends only on the fall height) is nearly the same for the two cases. The pile of fluid beneath the coil is taller at the higher frequency, because rope laid down more rapidly can mount higher before gravitational settling stops its ascent. The two coexisting states at a given total height therefore have slightly different values of the effective (corrected) fall height. Finally, the two states have opposite senses of rotation. This feature appears to be universal: for fall heights within the multivalued range, coiling switches back and forth repeatedly between low- and high-frequency states and changes its 


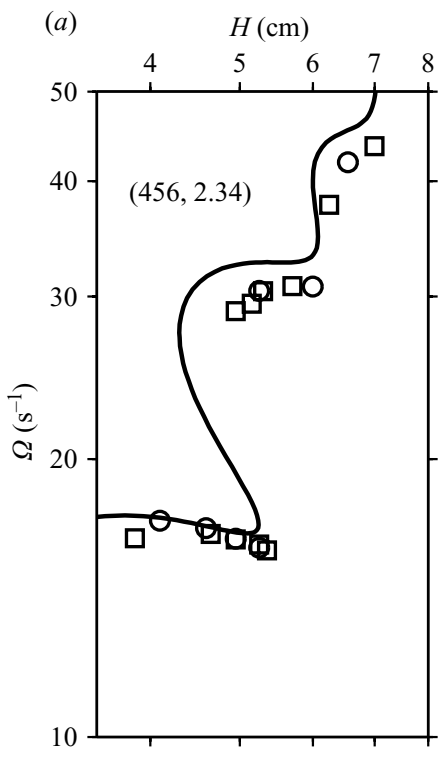

(c)

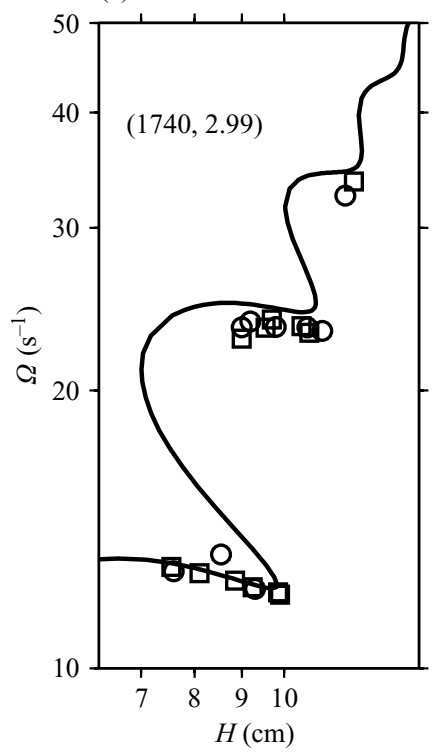

(d)

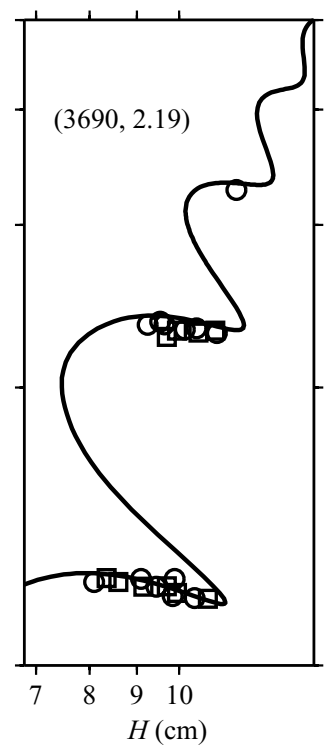

(b) $\quad H(\mathrm{~cm})$

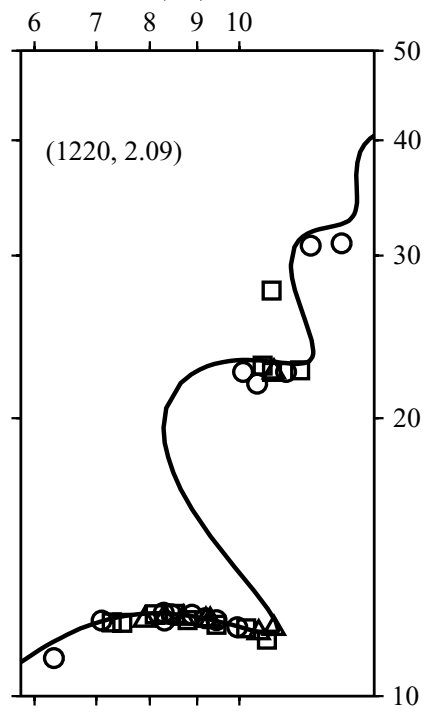

(e)

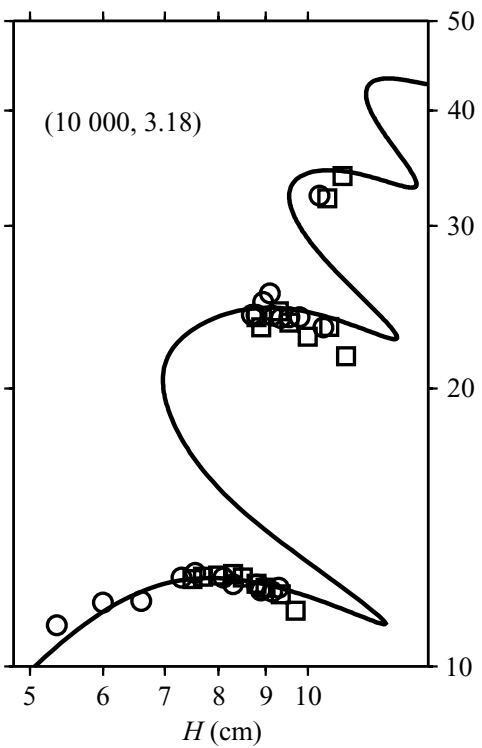

Figure 12. Comparison of experimentally measured (symbols) and numerically predicted (solid lines) frequencies as functions of height for the Zanjan experiments. The fluid viscosity was $v=300 \mathrm{~cm}^{2} \mathrm{~s}^{-1}$ for experiment $(a)$ and $1000 \mathrm{~cm}^{2} \mathrm{~s}^{-1}$ for experiments $(b)-(e)$. Values of $\left(\Pi_{1}, \Pi_{2}\right)$ for each experiment are indicated in parentheses. Measurements were obtained in series with $H$ increasing (squares), decreasing (circles), and varied randomly (triangles.) Error bars on $H$ are smaller than the size of the symbols. Error bars on $\Omega$, which in most cases do not exceed $\pm 5 \%$, have been omitted for clarity.

sense of rotation each time (this behaviour has also been reported by M. Maleki, private communication, 2004.) Each transition occurs via an intermediate state with a 'figure-of-eight' geometry (figure 13c). In general, the amplitude of the ' 8 ' is about the same as the diameter of the low-frequency coil (cf. figures $13 a$ and $13 c$ ). The smaller 

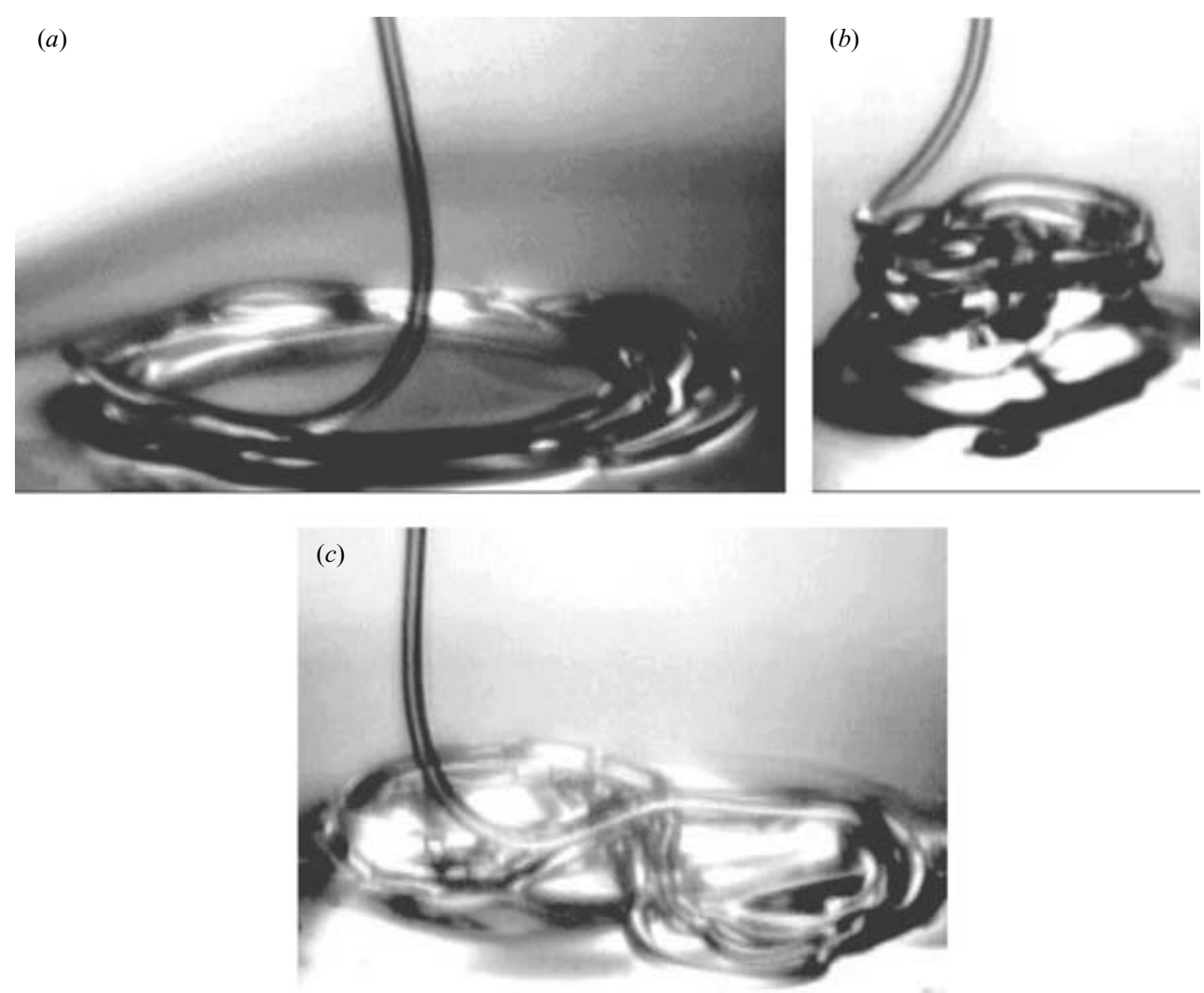

FIGURE 13. Geometry of coexisting coiling states in an experiment performed in Zanjan with $v=1000 \mathrm{~cm}^{2} \mathrm{~s}^{-1}, d=0.068 \mathrm{~cm}, Q=0.0042 \mathrm{~cm}^{3} \mathrm{~s}^{-1}\left(\Pi_{1}=6725, \Pi_{2}=3.76\right)$. The total (uncorrected) fall height is $7.1 \mathrm{~cm}$, and the radius of the portion of the rope shown is $0.028 \mathrm{~cm}$. (a) low-frequency state; $(b)$ high-frequency state; $(c)$ transitional 'figure-of-eight' state.

high-frequency coil is sometimes centred on the preceding ' 8 ', and sometimes forms over one of its loops. What triggers these transitions is not clear from our experiments; one possibility is irregularities in the pile of fluid beneath the coil (Maleki et al. 2004).

\section{Discussion}

The results obtained above allow us to construct a complete phase diagram for liquid rope coiling in the limit of strong gravitational stretching $\left(a_{1} \ll a_{0}\right)$ and $\Pi_{1} \equiv$ $\left(v^{5} / g Q^{3}\right)^{1 / 5} \gg 1$. The diagram is best understood when displayed in the form of a generic curve of the dimensionless frequency $\Omega\left(v / g^{2}\right)^{1 / 3} \equiv \hat{\Omega} v$ s. the dimensionless fall height $H\left(g / \nu^{2}\right)^{1 / 3} \equiv \hat{H}$. Figure 14 shows such a curve (calculated for the particular case $\Pi_{1}=10^{5}$ and $\Pi_{2}=0.562$ ), together with the important scalings that characterize it. For small heights, coiling is purely gravitational with negligible inertia. The scaling law for the frequency is $\Omega \sim \Omega_{G}$, which when rewritten using (3.5) takes the form $\hat{\Omega} \sim \hat{H}^{2} \Pi_{1}^{5 / 12}$. The next regime to be encountered is inertio-gravitational (IG) coiling, with a scaling law $\hat{\Omega} \sim \hat{H}^{-1 / 2}$. The transition from $G$ to IG coiling occurs at a value of $H$ where $\Omega_{G} \sim \Omega_{I G}$, or $\hat{H} \sim \Pi_{1}^{-1 / 6}$. IG coiling persists until $H$ reaches a value at which inertial forces in the coil become comparable to the viscous and gravitational forces there. That height is given by (3.12), which corresponds to $\hat{H} \sim \Pi_{1}^{-5 / 48}$. The 


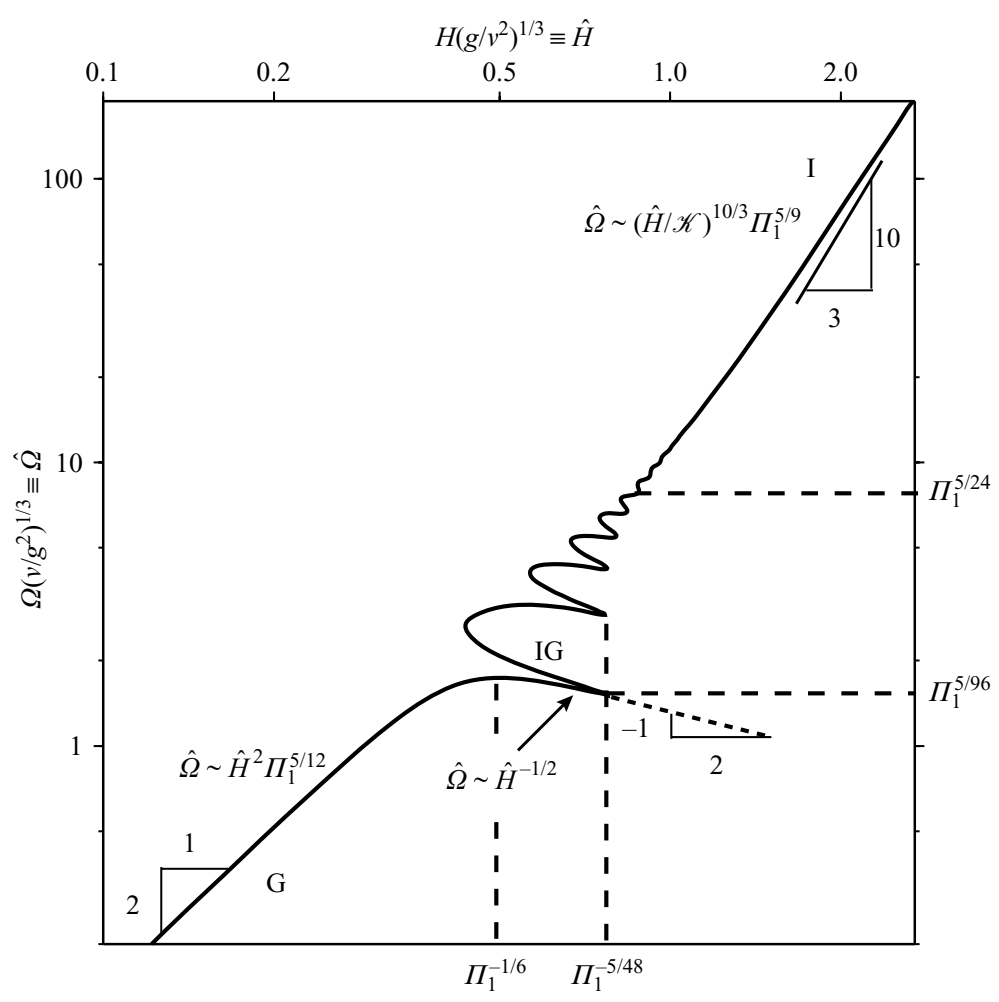

FIGURE 14. Phase diagram for liquid rope coiling in the asymptotic limit $\Pi_{1} \gg 1$. The solid line shows the coiling frequency as a function of height for a reference case with $\Pi_{1}=10^{5}$ and $\Pi_{2}=0.562$. The scaling laws for gravitational $(\mathrm{G})$, inertio-gravitational (IG), and inertial (I) coiling are indicated. The locations of the transitions between the laws are shown to order of magnitude by dashed lines. The function $\mathscr{K}(\hat{H})$ is shown in figure 15 .

rather narrow range of heights over which IG coiling occurs contrasts with the large ranges for the other three regimes.

Further increase in the fall height is now blocked while the rope passes through a series of 'whirling string' eigenstates, whose frequencies are shown in figure 8 . The number $N$ of such states that the coiling rope successively inhabits before purely inertial coiling sets in can be estimated by a simple argument. The frequency at turning point $n$ is $\Omega_{n} \sim\left(g^{2} / v\right)^{1 / 3} \Pi_{1}^{5 / 96} \tilde{\Omega}_{n}$, where $\tilde{\Omega}_{n}$ is the dimensionless eigenfrequency (figure 8). The corresponding radius of the contact point $s=\ell$ of the rope is $R_{n} \equiv$ $U_{1} / \Omega_{n}$, or equivalently

$$
R_{n} \sim\left(v^{2} / g\right)^{1 / 3} \Pi_{1}^{-25 / 96} \tilde{\Omega}_{n}^{-1}
$$

where we have used the relation $U_{1} \sim g H^{2} / v$ and the expression (3.12) for the height at the first turning point. According to (5.1), the radius $R_{n}$ decreases as the eigenfrequency increases. Eventually, $R_{n}$ becomes so small that it merges into the bending boundary layer near the contact point, whose arcwise extent is $\delta \sim(\nu Q / g)^{1 / 4}$. Because $\tilde{\Omega} \sim n$ for large $n$, the merging occurs after an eigenstate (or turning point) whose index and frequency scale as

$$
N \sim \Pi_{1}^{5 / 32}, \quad \hat{\Omega}_{N} \sim \Pi_{1}^{5 / 24}
$$




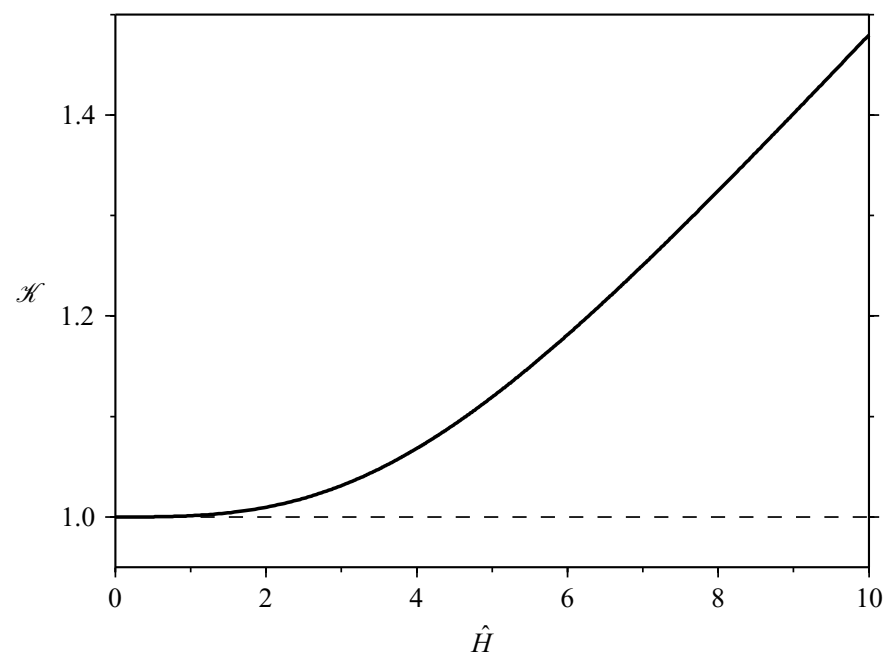

FIGURE 15. Function $\mathscr{K}(\hat{H})$ appearing in (5.3) that measures the inhibiting effect of axial inertia on gravitational stretching of the tail of the rope.

For frequencies exceeding $\Omega_{N}$, coiling is dominantly inertial. To understand the slope of the curve of frequency vs. height in this regime, it is necessary to note that the axial inertia $\rho A U U^{\prime}$ in the tail of the rope becomes significant for heights $H \sim\left(v^{2} / g\right)^{1 / 3}$. The generalization of (3.5) that includes this effect is (Maleki et al. 2004)

$$
a_{1}=\left(\frac{3 \pi \nu Q}{2 g}\right)^{1 / 2} H^{-1} \mathscr{K}(\hat{H})
$$

where the function $\mathscr{K}(\hat{H})$ is shown in figure 15 . The fact that $\mathscr{K}(\hat{H}) \geqslant 1$ indicates that inertia increases the radius $a_{1}$ by inhibiting the gravitational stretching of the tail. Upon substituting (5.3) into the scaling law $\Omega \sim \Omega_{I}$ for inertial coiling, we obtain

$$
\hat{\Omega}_{I} \sim\left[\frac{\hat{H}}{\mathscr{K}(\hat{H})}\right]^{10 / 3} \Pi_{1}^{5 / 9} .
$$

Because $\mathscr{K}$ noticeably exceeds unity for $\hat{H} \geqslant 1.5$ (figure 15 ), the slope of $\Omega(H)$ at the top right-hand side of figure 14 is slightly less than $10 / 3$.

The multiple 'spikes' in the scaled curves of frequency $v$ s. height in figure 9 strongly suggest that IG coiling may reflect a resonance phenomenon. Recall that the frequency of gravitational coiling is controlled by the dynamics in the 'coil' portion of the rope. Therefore if the frequency set by the coil happens to be close to an eigenfrequency of the tail, the coil will excite a resonant oscillation of the tail. Accordingly, the spikes in figure 9 can be interpreted as resonant oscillations that occur when $0.5 \Omega_{G} \approx \Omega_{n}$, where $0.5 \Omega_{G}$ is the frequency of gravitational coiling and $\Omega_{n}$ is one of the whirling string eigenfrequencies shown in figure 8.

An important problem remaining to be solved is that of the stability of our numerical solutions. The Zanjan experiments (figure 12) show that observable states of steady coiling in the multivalued regime are concentrated along the nearly horizontal steps in the curve of frequency vs. height below turning points. None of the steady states we observed lies on the steeply sloping switchback between the first two steps, and only in two cases (figure $12 a$ and 12b) did we observe states that may lie on 
the second (less steeply sloping) switchback. This suggests that states along the first switchback (at least) may be unstable to small perturbations. The rather complicated linear stability analysis required to answer this question is currently underway, and will be reported separately.

This research was supported by the Centre Nationale de la Recherche Scientifique (France). We thank A. Boudaoud, A. Davaille and J. Lister for helpful discussions, and H. Khalesifard, M. Maleki, S. N. Seyedreihani and R. Vafabakhsh for assistance with the experiments in Zanjan. The work in Zanjan was conducted in the laboratory of R. Golestanian, without whom these experiments would not have been possible. S. Decroocq of BP kindly provided data on the surface tension coefficient of HYVIS 30. Careful and constructive reviews by three anonymous referees helped greatly to improve the manuscript, and we are grateful to one of them for suggesting the 'resonance' interpretation of the multiple frequencies.

\section{Appendix. Model for a steadily whirling liquid string}

Consider a liquid string with density $\rho$ and dynamic viscosity $\mu$, injected at volumetric rate $Q$ from a circular hole of area $A_{0} \equiv \pi a_{0}^{2}$ and rotating with a steady angular velocity $\Omega$ about a vertical axis (figure $7 a$ ). Because the string can stretch, its cross-sectional area $A(s)$ and the fluid velocity $U(s) \equiv Q / A(s)$ along it are functions of the arclength $s$ measured from the injection point $s=0$. The string is assumed to be nearly vertical, and its total length $\ell \approx H$ is maintained constant by continual removal of the fluid that passes the cross-section $s=H$.

We assume that the string has negligible resistance to bending and twisting, so that its motion is governed by a balance among gravity, inertia and the axial tension associated with stretching. In the limit $\left(g H^{3} / v^{2}\right)^{1 / 2} \ll 1$, both the Coriolis force and the axial inertia $\rho A U\left(U \boldsymbol{d}_{3}\right)^{\prime}$ are negligible, and inertia is dominantly centrifugal. The components of the motion of the string in orthogonal horizontal directions are then decoupled, and the string can be regarded, with no loss of generality, as being confined to a plane. Let $y(s)$ be the lateral displacement of the string from the vertical. Define $\boldsymbol{e}_{i}(i=1,2,3)$ to be Cartesian unit vectors in a reference frame rotating with the string, such that $\boldsymbol{e}_{3}$ points downward and $\boldsymbol{e}_{1}$ lies in the plane of the string. Let $\boldsymbol{d}_{i}(s)$ $(i=1,2,3)$ be orthogonal unit vectors defined at each point on the axis of the string, such that $\boldsymbol{d}_{3}$ is tangent to the axis and $\boldsymbol{d}_{1}$ lies in the plane of the string. Because the string is nearly vertical,

$$
\boldsymbol{d}_{1} \sim \boldsymbol{e}_{1}-y^{\prime} \boldsymbol{e}_{3}, \quad \boldsymbol{d}_{2}=\boldsymbol{e}_{2}, \quad \boldsymbol{d}_{3} \sim \boldsymbol{e}_{3}+y^{\prime} \boldsymbol{e}_{1},
$$

where primes denote differentiation with respect to $s$. The rate of change of the tangent vector $\boldsymbol{d}_{3}$ along the axis of the string is

$$
\boldsymbol{d}_{3}^{\prime}=y^{\prime \prime} \boldsymbol{d}_{1} \text {. }
$$

The equation governing the motion of the string is

$$
\left(N \boldsymbol{d}_{3}\right)^{\prime}+\rho g A \boldsymbol{e}_{3}=\rho A \Omega^{2} y \boldsymbol{e}_{2},
$$

where

$$
N=3 \mu A U^{\prime}
$$

is the axial viscous force and $3 \mu$ is the extensional viscosity. For a nearly vertical string, the component of the centrifugal force in the axial $\left(\boldsymbol{d}_{3^{-}}\right)$direction is negligible. 
The dominant force balance in that direction is therefore between gravity and viscous resistance to stretching, or

$$
0=N^{\prime}+\rho g A .
$$

An analytical solution of (A 5) and (A 4) subject to the boundary conditions $U(0)-$ $U_{0}=U^{\prime}(H)=0$ was obtained by Ribe (2004), and is

$$
U=\frac{2}{3 k^{2}} \frac{g H^{2}}{v} \cos ^{2} \frac{k(H-s)}{2 H},
$$

where $k$ satisfies the transcendental equation

$$
0=2 B \cos ^{2} \frac{1}{2} k-3 k^{2}
$$

and $B=g H^{2} / \nu U_{0}$. In the limit $B \gg 1$ that corresponds to strong stretching $\left(A(H) \ll A_{0}\right)$ of the string,

$$
k \sim \pi\left[1-\sqrt{6} B^{-1 / 2}+6 B^{-1}+O\left(B^{-3 / 2}\right)\right] .
$$

Projecting the force balance (A 3 ) onto the lateral basis vector $\boldsymbol{d}_{1}$ and making use of the solution (A 6) for $U$, we obtain

$$
0=\frac{g H}{k} \sin \frac{k(H-s)}{H} y^{\prime \prime}-g y^{\prime}+\Omega^{2} y .
$$

The first term in (A 9) is proportional to the viscous force normal to the axis that is generated by the stretching of a curved string. In the limit $k \rightarrow 0$, (A 9) reduces to the classical equation governing the motion of a whirling inextensible string (Antman 1995). Because (A 9) has a regular singular point at $s=H$, the appropriate boundary conditions are $y(0)=0$ and the requirement that $y(H)$ be finite.

\section{REFERENCES}

Antman, S. 1995 Nonlinear Problems of Elasticity. Springer.

Barnes, G. \& MacKenzie, R. 1959 Height of fall versus frequency in liquid rope-coil effect. Am. $J$. Phys. 27, 112-115.

Barnes, G. \& Woodcock, R. 1958 Liquid rope-coil effect. Am. J. Phys. 26, 205-209.

Brenner, M. \& Parachuri, S. 2003 Thermal bending of liquid sheets and jets. Phys. Fluids 15, $3568-3571$.

Cruickshank, J. O. 1980 Viscous fluid buckling: a theoretical and experimental analysis with extensions to general fluid stability. PhD thesis, Iowa State University, Ames, Iowa.

Cruickshank, J. O. 1988 Low-Reynolds-number instabilities in stagnating jet flows. J. Fluid Mech. 193, 111-127.

Cruickshank, J. O. \& Munson, B. R. 1981 Viscous fluid buckling of plane and axisymmetric jets. J. Fluid Mech. 113, 221-239.

Doedel, E., Champneys, A. R., Fairgrieve, T. F., Kuznetsov, Y. A., Sandstede, B. \& Wang, X. 2002 AUTO 97: Continuation and bifurcation software for ordinary differential equations. http://indy.cs.concordia.ca/auto/.

Griffiths, R. W. \& TURneR, J. S. 1988 Folding of viscous plumes impinging on a density or viscosity interface. Geophys. J. 95, 397-419.

Huppert, H. E. 1986 The intrusion of fluid mechanics into geology. J. Fluid Mech. 173, 557-594.

Mahadevan, L., Ryu, W. S. \& Samuel, A. D. T. 1998 Fluid 'rope trick' investigated. Nature 392, 140.

Mahadevan, L., Ryu, W. S. \& Samuel, A. D. T. 2000 Correction: fluid 'rope trick' investigated. Nature 403, 502 . 
Maleki, M., Habibi, M., Golestanian, R., Ribe, N. M. \& Bonn, D. 2004 Liquid rope coiling on a solid surface. Phys. Rev. Lett. 93, 214502.

Ribe, N. M. 2004 Coiling of viscous jets. Proc. R. Soc. Lond. A 460, 3223-3239.

SkorobogatiY, M. \& Mahadevan, L. 2000 Folding of viscous sheets and filaments. Europhys. Lett. 52, 532-538.

TAYLOR, G. I. 1968 Instability of jets, threads, and sheets of viscous fluid. Proc. Intl Congr. Appl. Mech. Springer.

Tchavdarov, B., Yarin, A. L. \& Radev, S. 1993 Buckling of thin liquid jets. J. Fluid Mech. 253, 593-615. 